\title{
Association of estimated dietary acid load with albuminuria in Japanese adults: a cross-sectional study
}

\author{
Keiko Kabasawa ${ }^{1 *}$ (D), Michihiro Hosojima ${ }^{2}$, Ribeka Takachi ${ }^{3}$, Kazutoshi Nakamura ${ }^{4}$, Yumi Ito $^{1}$, Akihiko Saito ${ }^{5}$,
} Norie Sawada ${ }^{6}$, Shoichiro Tsugane ${ }^{6}$, Junta Tanaka ${ }^{1}$ and Ichiei Narita ${ }^{1,7}$

\begin{abstract}
Background: Acid-base imbalance might promote the progression of chronic kidney disease (CKD), but whether nutrient-derived dietary acid load increases the risk of albuminuria or even high normoalbuminuria is unclear.

Methods: A Japanese cohort comprising 3250 men and 3434 women aged 40-97 years with urine albumin-tocreatinine ratio $(A C R)<33.9 \mathrm{mg} / \mathrm{mmol}$ or estimated glomerular filtration rate $\geq 15 \mathrm{ml} / \mathrm{min} / 1.73 \mathrm{~m}^{2}$ were assessed. We performed a cross-sectional evaluation of the association between net endogenous acid production (NEAP), estimated as dietary protein to potassium content ratio, and the presence of high normoalbuminuria (ACR: 1.13-3. $38 \mathrm{mg} / \mathrm{mmol}$ ) or microalbuminuria.

Results: Median NEAP was 43.4 (interquartile range (IQR): 34.2-53.4) mEq/day in men and 35.0 (IQR: 27.7-43.6) mEq/ day in women. Median ACR was 1.11 (IQR: 0.57-2.49) mg/mmol in men and 1.47 (IQR: 0.82-2.83) mg/mmol in women. In multivariate analysis, the adjusted odds ratio of the highest versus lowest NEAP quartile for microalbuminuria was 1. 47 (95\% confidence interval (Cl): 1.08-1.99) in men and 1.54 (95\% Cl: 1.11-2.14) in women. For high normoalbuminuria or microalbuminuria, the adjusted odds ratio was 1.28 (95\% Cl: 1.02-1.59) in men and 1.39 (95\% Cl: 1.11-1.74) in women. From nutrient composition analysis, subjects with the highest potassium intake, but not protein intake, had lower adjusted odds ratios for the presence of microalbuminuria than those in the lowest quartile for potassium intake.
\end{abstract}

Conclusions: Higher NEAP was associated with albuminuria and its association might negatively relate to potassium intake in an adult Japanese population.

Keywords: Acid-base imbalance, Albuminuria, East Asian, Nutrition, Potassium

\section{Background}

An association between acid-base imbalance and kidney disease has been suggested in recent decades [1, 2]. Acid-base balance is mainly controlled by kidney function, but could be also affected by the intake of acid-inducing foods $[2,3]$. Several studies have reported an association between acid-base imbalance and chronic kidney disease (CKD) using albuminuria including microalbuminuria as an indicator [4-6]. Albuminuria is a known risk factor for cardiovascular disease and all-cause mortality. Recently,

\footnotetext{
* Correspondence: keikoyk@med.niigata-u.ac.jp

${ }^{1}$ Department of Health Promotion Medicine, Niigata University Graduate School of Medical and Dental Sciences, 1-757 Asahimachi-dori, Chuo-ku, Niigata 951-8510, Japan

Full list of author information is available at the end of the article
}

high normoalbuminuria (urine albumin-to-creatinine ratio $[\mathrm{ACR}]>1.13 \mathrm{mg} / \mathrm{mmol}(10 \mathrm{mg} / \mathrm{g}))$ has also been suggested to carry a similar risk in the general population [7-9]. Thus, detection of high normoalbuminuria, as well as microalbuminuria, is important for the prevention of CKD, cardiovascular events, and death. However, no previous studies have demonstrated the association between acidbase balance and high normoalbuminuria.

The estimated dietary acid load is derived from an equation that takes into account organic compounds and is referred to as net endogenous acid production (NEAP). NEAP, estimated by the ratio of dietary protein to potassium content, reflects the balance of acid and base precursors in healthy individuals in a steady state [10] and in individuals with CKD [11]. Lower dietary

(C) The Author(s). 2019 Open Access This article is distributed under the terms of the Creative Commons Attribution 4.0 International License (http://creativecommons.org/licenses/by/4.0/), which permits unrestricted use, distribution, and 
protein intake was shown to be associated with reduction in NEAP [12], and consequently protein has been considered as the major source of nonvolatile acids due to its metabolism to sulfates and other organic acids [13]. Nevertheless, some studies have reported that protein intake alone was less likely to be associated with CKD in terms of the relationship between NEAP and CKD [6, 14]. Moreover, the association between albuminuria and nutrients is not fully understood.

In this context, intake of other nutrients such as potassium, as well as protein, should be reassessed in relation to NEAP and albuminuria in various settings. Hence, this study sought to determine the association of estimated dietary acid load with microalbuminuria and/or high normoalbuminuria, and to evaluate the association between nutrient components in estimated dietary acid load and albuminuria.

\section{Methods}

\section{Study population}

This cross-sectional study is based on baseline medical examination findings of the Uonuma CKD Cohort Study, which was a population-based prospective cohort study conducted between 2012 and 2015 in the Uonuma region of Niigata Prefecture, Japan, comprising Minamiuonuma City, Uonuma City, and Yuzawa Town [15]. For baseline medical examinations, all 11,406 residents underwent annual local health-check examinations and 8052 of them were subjected to biochemical sampling; 6950 provided urine samples and completed a lifestyle-related questionnaire. We excluded subjects who had low estimated glomerular filtration rate (eGFR) values $(<15$ $\mathrm{ml} / \mathrm{min} / 1.73 \mathrm{~m}^{2}$ ), macroalbuminuria (ACR $\geq 33.9 \mathrm{mg} /$ $\mathrm{mmol}$ ), and those who had missing values or incomplete questionnaire data (Fig. 1). Finally, the total number of subjects for analysis in this study was 6684 comprising 3250 men and 3434 women.

\section{Data collection}

During the baseline survey, each participant underwent a health-check examination in the morning or afternoon with or without fasting. The medical examination assessed body weight, body height, blood pressure, fasting or casual plasma glucose, serum creatinine, and glycated hemoglobin (HbA1c), and urine measurements, including urine ACR. Also, self-reported information on antihypertensive or antidiabetic medication was obtained.

Body mass index (BMI) was calculated as body weight $(\mathrm{kg})$ divided by height squared $\left(\mathrm{m}^{2}\right)$. Blood pressure was measured once or twice by using a pressurized cuff on the upper arm at rest in the sitting position. Pulse pressure was calculated as systolic blood pressure minus diastolic blood pressure. Blood glucose was measured by the hexokinase method and HbA1c was measured using high-performance liquid chromatography. Serum creatinine concentration was measured by the enzymatic method. eGFR was obtained by using the following formula modified for Japanese adults [16]: eGFR ( $\mathrm{ml} / \mathrm{min} /$ $\left.1.73 \mathrm{~m}^{2}\right)=194 \times[\text { Serum creatinine }(\mathrm{mg} / \mathrm{dl})]^{-1.094} \times($ Age $)^{-0.287} \times 0.739$ (for women). Diabetes was diagnosed

Uonuma CKD cohort study; Participants of biochemical sampling (health-check examination)

$$
\mathrm{N}=8052
$$

Participants of both questionnaire and biochemical sampling (health-check examination)

$$
\mathrm{N}=6950
$$

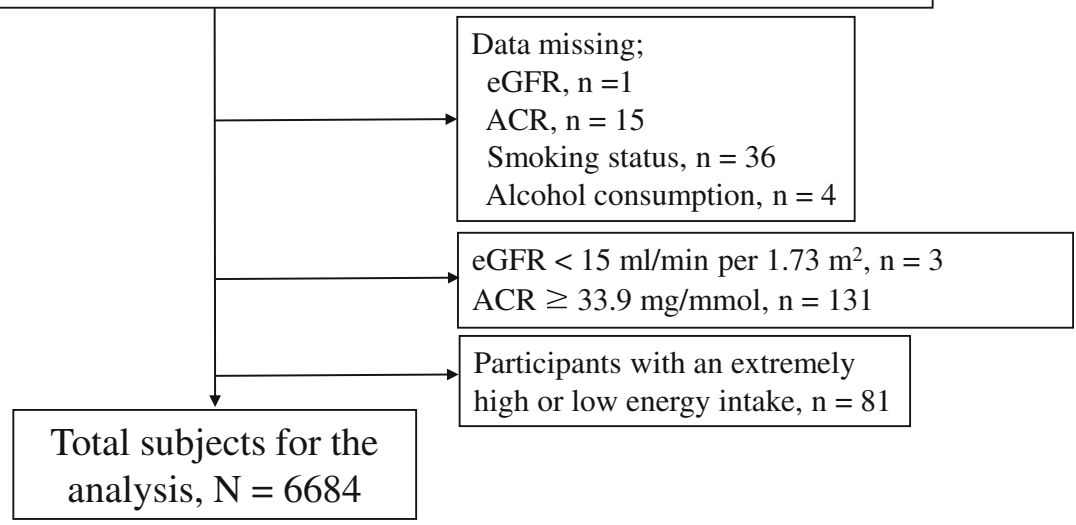

Fig. 1 Flowchart of included participants 
based on HbA1c $\geq 6.5 \%$ and fasting plasma glucose $\geq 7.0$ $\mathrm{mmol} / \mathrm{l}$ or casual plasma glucose $\geq 11.1 \mathrm{mmol} / \mathrm{l}$, or taking antidiabetic medication. Hypertension was defined as systolic blood pressure $\geq 140 \mathrm{mmHg}$ or diastolic blood pressure $\geq 90 \mathrm{mmHg}$ in accordance with the World Health Organization criteria [17] or alternatively, taking antihypertensive medication.

Urine albumin concentration was measured by the latex agglutination method, and urine creatinine concentration was measured by the enzymatic method in spot urine samples. Albuminuria was evaluated as ACR, which was calculated as urine albumin concentration divided by urinary creatinine concentration. Using ACR cutoff values suggested in a previous study [18], "high normoalbuminuria", "microalbuminuria", and "high normoalbuminuria or microalbuminuria" were defined as ACR $1.13-3.38 \mathrm{mg} / \mathrm{mmol}$ $(10.0-29.9 \mathrm{mg} / \mathrm{g}), \quad 3.39-33.8 \mathrm{mg} / \mathrm{mmol} \quad(30.0-299 \mathrm{mg} / \mathrm{g})$, and $1.13-33.8 \mathrm{mg} / \mathrm{mmol}(10.0-299 \mathrm{mg} / \mathrm{g})$, respectively.

\section{Assessment of lifestyle and dietary intake}

Demographic characteristics, smoking habit, alcohol consumption, physical activity, and food consumption data were obtained from a self-administered questionnaire. A summary of smoking habit, alcohol consumption, and total physical activity can be extracted from the questionnaire [19]. Dietary assessment was based on a validated food frequency questionnaire (FFQ) [20]. For the validation, Spearman's rank correlation coefficients were calculated for energy-adjusted values between intakes based on the FFQ and 12-day weighed food records. Spearman's rank correlation coefficients for protein and potassium intake were 0.40 and 0.48 in men and 0.33 and 0.54 in women, respectively.

Estimated dietary acid load was evaluated using the NEAP and the potential renal acid load (PRAL). NEAP and PRAL were derived using a previously published equation: $\operatorname{NEAP}(\mathrm{mEq} /$ day $)=54.5 \times$ protein $(\mathrm{g} /$ day $) /$ potassium $(\mathrm{mEq} /$ day) -10.2 [10]. PRAL $(\mathrm{mEq} /$ day $)=0.4888 \times$ protein intake $(\mathrm{g} /$ day $)+0.0366 \times$ phosphorus $(\mathrm{mg} /$ day $)-0.0205 \times$ potassium (mg/day) $-0.0125 \times$ calcium $(\mathrm{mg} /$ day $)-0.0263 \times$ magnesium (mg/day) [21]. Energy-adjusted intakes of protein, potassium, and other specified nutrients or food groups were determined by the residual method [22] after excluding subjects with extreme energy intake $(>$ or $<3$ standard deviations [SD] from the mean). NEAP and PRAL estimated from these equations have been validated against based on 24-h urine samples in adolescents and adults [12, 23]. The detailed procedure of the questionnaire survey has been described elsewhere [15, 24].

\section{Statistical analysis}

Characteristics of the subjects are presented as means \pm $\mathrm{SD}$, medians (interquartile range [IQR]) or numbers (percentages). Differences in characteristics between men and women were analyzed using the Wilcoxon rank-sum test for continuous variables and the chi-squared test for categorical variables. The unadjusted trend association between NEAP quartile and covariates including potential confounding variables was tested for by using the linear regression model for continuous covariates or the logistic regression model for categorical covariates (yes/no) assigning the NEAP quartile as a continuous variable (Table 1).

Multivariate logistic regression analysis was performed to calculate adjusted odds ratios and 95\% confidence intervals (95\% CI) for each grade of albuminuria. In these calculations, "Controls" are normoalbuminuria cases (ACR $<1.13$ $\mathrm{mg} / \mathrm{mmol}$ ) (Tables 2 and 3). Adjustments were done with potential confounders in three models as follows: Model 1 is adjusted for only age (years, continuous); Model 2 is adjust as for Model 1 plus BMI ( $\mathrm{kg} / \mathrm{m}^{2}$, continuous), physical activity (metabolic equivalent task hour, continuous), smoking status (never-smoker, former smoker, or current smoker), alcohol consumption (<150 g, 150-299 g, 300$449 \mathrm{~g},>450 \mathrm{~g}$ ethanol/week), and energy intake ( $\mathrm{kcal} /$ day, quartile); and Model 3 is adjusted as for Model 2 plus eGFR $\left(\mathrm{ml} / \mathrm{min} / 1.73 \mathrm{~m}^{2}\right.$, continuous), pulse pressure ( $\mathrm{mmHg}$, continuous), dietary salt intake (g/day, continuous), diabetes (yes or no), and hypertension (yes or no). For sensitivity analysis, we performed a similar multivariate logistic regression analysis to calculate odds ratios of each grade of albuminuria according to PRAL quartile. Furthermore, to examine the role of the nutrient components of NEAP for albuminuria, the association between dietary protein (including animal and plant protein, respectively) or potassium intake and albuminuria was assessed by a similar multivariate logistic regression analysis, as described above. All analyses were performed with SAS version 9.4 (SAS Institute Inc., Cary, NC).

\section{Results}

Subjects were 991 men (30.5\%) and 1451 women (42.2\%) with high normoalbuminuria and 630 men (19.4\%) and 705 women (20.5\%) with microalbuminuria. Mean \pm SD age was $69.2 \pm 10.3$ years in men and $68.3 \pm$ 9.6 years in women; median (IQR) NEAP was 43.4 $(34.2-53.4) \mathrm{mEq} /$ day in men and $35.0(27.7-43.6) \mathrm{mEq} /$ day in women; median ACR (IQR) was $1.11(0.57-2.49)$ $\mathrm{mg} / \mathrm{mmol}$ in men and $1.47(0.82-2.83) \mathrm{mg} / \mathrm{mmol}$ in women; and median eGFR (IQR) was 73.7 (64.1-84.3) $\mathrm{ml} / \mathrm{min}$ per $1.73 \mathrm{~m}^{2}$ in men and $73.7(64.7-83.7) \mathrm{ml} / \mathrm{min}$ per $1.73 \mathrm{~m}^{2}$ in women, respectively. Compared with women, men were older, were more frequently smokers, drinkers, hypertensive, and diabetic, had higher body mass index, total physical activity, systolic blood pressure, and diastolic blood pressure $(P<0.0001$, respectively), and had lower pulse pressure $(P=0.0015)$. For dietary content, meat and egg intake did not differ between men and women. 


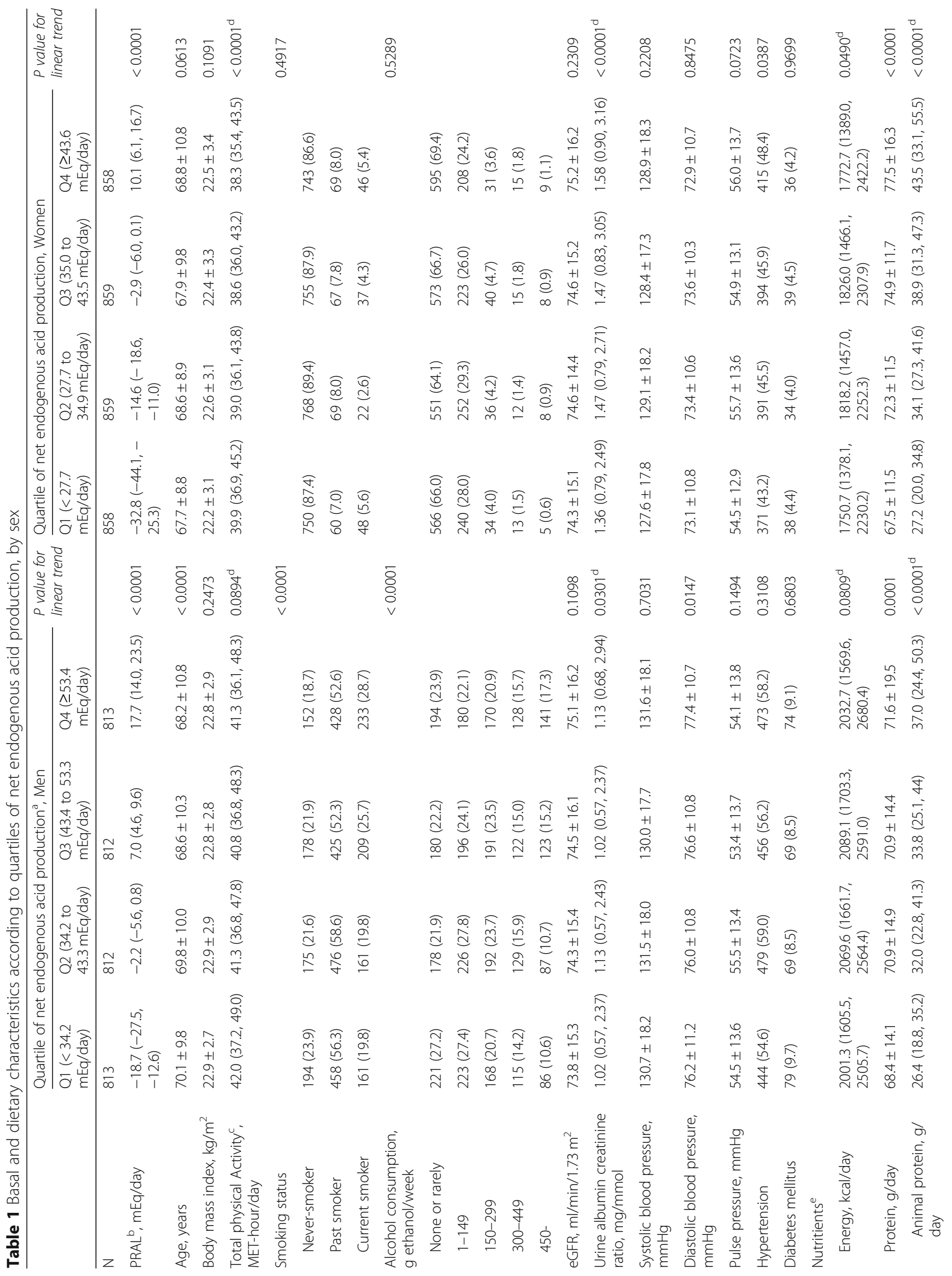




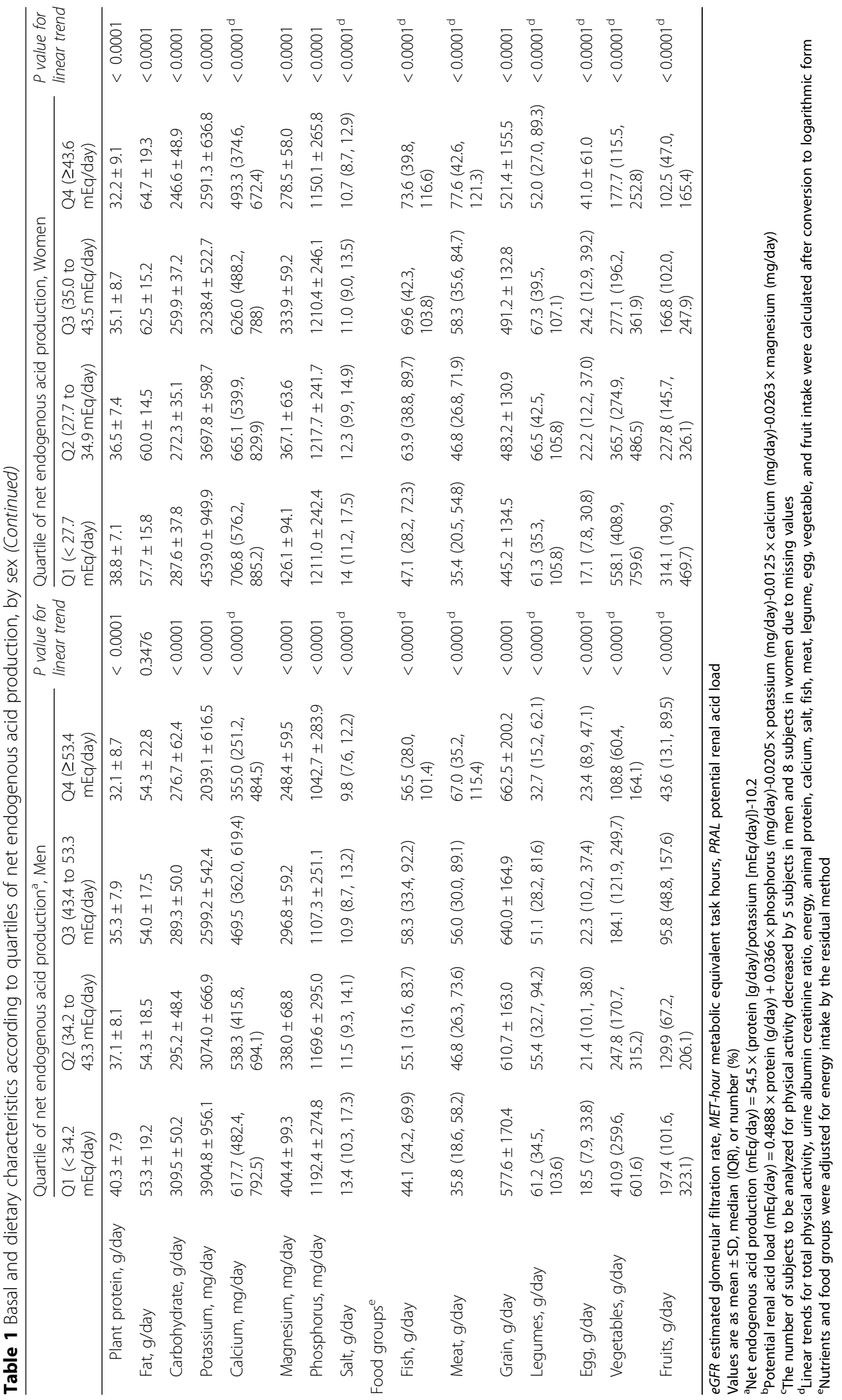


Table 2 Odds ratios (95\% Cls) for net endogenous acid production and risk of microalbuminuria (a), high normoalbuminuria (b), and high normoalbuminuria or microalbuminuria (c)

\begin{tabular}{|c|c|c|c|c|c|}
\hline (a) & \multicolumn{4}{|c|}{ Quartile of net endogenous acid production } & \multirow[t]{2}{*}{ P for trend } \\
\hline Men & Q1 (<34.0 mEq/day) & Q2 (34.0 to $43.4 \mathrm{mEq} /$ day) & Q3 (43.5 to $53.3 \mathrm{mEq} /$ day) & Q4 ( $\geq 53.4 \mathrm{mEq} /$ day) & \\
\hline Cases, $\mathrm{n}$ & 147 & 154 & 151 & 178 & \\
\hline Control, n & 417 & 411 & 415 & 386 & \\
\hline Unadjusted & 1.00 (reference) & 1.06 (0.82 to 1.38$)$ & 1.03 (0.79 to 1.35$)$ & 1.31 (1.01 to 1.69$)$ & 0.0598 \\
\hline Model 1 & 1.00 (reference) & $1.11(0.84$ to 1.46$)$ & 1.14 (0.87 to 1.50$)$ & 1.49 (1.14 to 1.95$)$ & 0.0047 \\
\hline Model 2 & 1.00 (reference) & $1.11(0.84$ to 1.47$)$ & 1.15 (0.87 to 1.53$)$ & $1.47(1.12$ to 1.94$)$ & 0.0074 \\
\hline Model 3 & 1.00 (reference) & $1.12(0.83$ to 1.50$)$ & $1.19(0.88$ to 1.61$)$ & $1.47(1.08$ to 1.99$)$ & 0.0130 \\
\hline Women & Q1 (<27.7 mEq/day) & Q2 (27.7 to $34.8 \mathrm{mEq} /$ day) & Q3 (34.9 to $43.1 \mathrm{mEq} /$ day) & Q4 ( $\geq 43.2 \mathrm{mEq} /$ day) & \\
\hline Cases, $\mathrm{n}$ & 142 & 171 & 187 & 205 & \\
\hline Control, n & 354 & 324 & 309 & 291 & \\
\hline Unadjusted & 1.00 (reference) & $1.32(1.01$ to 1.72$)$ & 1.51 (1.16 to 1.97$)$ & 1.76 (1.35 to 2.29) & $<0.0001$ \\
\hline Model 1 & 1.00 (reference) & $1.20(0.90$ to 1.60$)$ & 1.56 (1.18 to 2.08$)$ & 1.60 (1.20 to 2.13$)$ & 0.0003 \\
\hline Model 2 & 1.00 (reference) & 1.14 (0.85 to 1.52$)$ & 1.54 (1.15 to 2.06$)$ & $1.57(1.18$ to 2.11$)$ & 0.0004 \\
\hline Model 3 & 1.00 (reference) & 1.09 (0.80 to 1.50$)$ & 1.65 (1.19 to 2.27$)$ & $1.54(1.11$ to 2.14$)$ & 0.0014 \\
\hline (b) & \multicolumn{4}{|c|}{ Quartile of net endogenous acid production } & $P$ for trend \\
\hline Men & Q1 (<34.0 mEq/day) & Q2 (34.0 to $43.0 \mathrm{mEq} /$ day) & Q3 (43.1 to $53.0 \mathrm{mEq} /$ day) & Q4 ( $\geq 53.1 \mathrm{mEq} /$ day) & \\
\hline Cases, $\mathrm{n}$ & 238 & 258 & 239 & 256 & \\
\hline Control, n & 417 & 397 & 416 & 399 & \\
\hline Unadjusted & 1.00 (reference) & 1.14 (0.91 to 1.42$)$ & 1.01 (0.80 to 1.26$)$ & $1.12(0.90$ to 1.41$)$ & 0.5284 \\
\hline Model 1 & 1.00 (reference) & $1.15(0.92$ to 1.45$)$ & 1.07 (0.85 to 1.35$)$ & 1.22 (0.97 to 1.53 ) & 0.1642 \\
\hline Model 2 & 1.00 (reference) & 1.15 (0.91 to 1.44$)$ & 1.06 (0.84 to 1.34$)$ & 1.19 (0.95 to 1.50$)$ & 0.2190 \\
\hline Model 3 & 1.00 (reference) & 1.13 (0.89 to 1.43$)$ & 1.06 (0.83 to 1.35$)$ & 1.18 (0.93 to 1.51$)$ & 0.2391 \\
\hline Women & Q1 (<27.2 mEq/day) & Q2 (27.2 to $34.6 \mathrm{mEq} /$ day) & Q3 (34.7 to $43.2 \mathrm{mEq} /$ day) & Q4 ( $\geq 43.2$ mEq/day) & \\
\hline Cases, n & 344 & 355 & 359 & 393 & \\
\hline Control, n & 338 & 327 & 324 & 289 & \\
\hline Unadjusted & 1.00 (reference) & 1.07 (0.86 to 1.32$)$ & 1.09 (0.88 to 1.35$)$ & 1.34 (1.08 to 1.65$)$ & 0.0099 \\
\hline Model 1 & 1.00 (reference) & 1.05 (0.85 to 1.31$)$ & 1.11 (0.89 to 1.38 ) & $1.32(1.06$ to 1.65$)$ & 0.0124 \\
\hline Model 2 & 1.00 (reference) & 1.04 (0.83 to 1.29$)$ & 1.10 (0.88 to 1.37$)$ & $1.32(1.06$ to 1.65$)$ & 0.0130 \\
\hline Model 3 & 1.00 (reference) & 1.06 (0.84 to 1.33$)$ & 1.13 (0.89 to 1.43 ) & 1.34 (1.05 to 1.70$)$ & 0.0163 \\
\hline (c) & \multicolumn{4}{|c|}{ Quartile of net endogenous acid production } & $P$ for trend \\
\hline Men & Q1 (<34.2 mEq/day) & Q2 (34.2 to $43.3 \mathrm{mEq} /$ day) & Q3 (43.4 to $53.3 \mathrm{mEq} /$ day) & Q4 ( $\geq 53.4$ mEq/day) & \\
\hline Cases, n & 393 & 411 & 391 & 426 & \\
\hline Control, n & 420 & 401 & 421 & 387 & \\
\hline Unadjusted & 1.00 (reference) & $1.10(0.90$ to 1.33$)$ & $0.99(0.82$ to 1.21$)$ & 1.18 (0.97 to 1.43$)$ & 0.2155 \\
\hline Model 1 & 1.00 (reference) & 1.11 (0.91 to 1.36$)$ & 1.07 (0.87 to 1.30$)$ & $1.30(1.07$ to 1.59$)$ & 0.0212 \\
\hline Model 2 & 1.00 (reference) & 1.11 (0.91 to 1.36$)$ & 1.06 (0.87 to 1.30$)$ & $1.27(1.04$ to 1.56$)$ & 0.0414 \\
\hline Model 3 & 1.00 (reference) & 1.09 (0.88 to 1.35$)$ & 1.09 (0.88 to 1.35$)$ & $1.28(1.02$ to 1.59$)$ & 0.0407 \\
\hline Women & Q1 (<27.7 mEq/day) & Q2 (27.7 to $34.9 \mathrm{mEq} /$ day) & Q3 (35.0 to $43.6 \mathrm{mEq} /$ day) & Q4 ( $\geq 43.7 \mathrm{mEq} /$ day) & \\
\hline Cases, $\mathrm{n}$ & 504 & 533 & 541 & 578 & \\
\hline Control, n & 354 & 326 & 318 & 280 & \\
\hline Unadjusted & 1.00 (reference) & 1.15 (0.95 to 1.39$)$ & 1.20 (0.98 to 1.45$)$ & 1.45 (1.19 to 1.77$)$ & 0.0003 \\
\hline
\end{tabular}


Table 2 Odds ratios (95\% Cls) for net endogenous acid production and risk of microalbuminuria (a), high normoalbuminuria (b), and high normoalbuminuria or microalbuminuria (c) (Continued)

\begin{tabular}{llllll}
\hline Model 1 & 1.00 (reference) & $1.10(0.90$ to 1.34$)$ & $1.20(0.98$ to 1.47$)$ & $1.41(1.15$ to 1.74$)$ & 0.0007 \\
Model 2 & 1.00 (reference) & $1.07(0.87$ to 1.31$)$ & $1.19(0.97$ to 1.45$)$ & $1.40(1.14$ to 1.72$)$ & 0.0010 \\
Model 3 & 1.00 (reference) & $1.08(0.87$ to 1.33$)$ & $1.21(0.97$ to 1.50$)$ & $1.39(1.11$ to 1.74$)$ & 0.0028 \\
\hline
\end{tabular}

Net endogenous acid production $=54.5 \times$ protein $(\mathrm{g} /$ day $) /$ potassium $(\mathrm{mEq} /$ day $)-10.2$

'Controls' means normoalbuminuric cases ( $A C R<1.13 \mathrm{mg} / \mathrm{mmol}$ )

Model 1 was adjusted for age (years, continuous); Model 2 was adjusted as for model 1 plus body mass index (kg/m², continuous), physical activity (metabolic equivalent task hour, continuous), smoking status (never-smoker, former smoker, or current smoker), alcohol consumption (<150 g, $150-299 \mathrm{~g}, 300-449 \mathrm{~g},>450 \mathrm{~g}$ ethanol/week), energy intake(kcal/day, quartiles); and Model 3 was adjusted as for model 2 plus eGFR ( $\mathrm{ml} / \mathrm{min} / 1.73 \mathrm{~m}{ }^{2}$, continuous), dietary salt intake (g/day, continuous), pulse pressure ( $\mathrm{mmHg}$, continuous), diabetes (yes or no), and hypertention (yes or no)

In model 2 and model 3, the number of subjects to be analyzed decreased by 4 men and 3 women for (a), for 4 men and 7 women (b) and 5 men and 8 women for (c) due to missing values for physical activity

Table 3 Odds ratios (95\% Cls) for protein (a) or potassium (b) intake and risk of microalbuminuria

\begin{tabular}{|c|c|c|c|c|c|}
\hline (a) & Quartile of protein intake & & & & $P$ for trend \\
\hline Men & Q1 (<60.3 g/day) & Q2 (60.3 to $69.6 \mathrm{~g} /$ day) & Q3 (69.7 to 78.5 g/day) & Q4 ( $\geq 78.6$ g/day) & \\
\hline Cases, n & 146 & 156 & 161 & 167 & \\
\hline Controls, n & 418 & 410 & 403 & 398 & \\
\hline Unadjusted & 1.00 (reference) & 1.09 (0.84 to 1.42$)$ & 1.14 (0.88 to 1.49$)$ & 1.20 (0.93 to 1.56$)$ & 0.1554 \\
\hline Model 1 & 1.00 (reference) & $0.93(0.71$ to 1.23$)$ & 0.90 (0.68 to 1.18$)$ & 0.83 (0.63 to 1.09) & 0.1738 \\
\hline Model 2 & 1.00 (reference) & 1.09 (0.82 to 1.45$)$ & 1.09 (0.81 to 1.47$)$ & 1.01 (0.74 to 1.38$)$ & 0.9946 \\
\hline Model 3 & 1.00 (reference) & 1.11 (0.82 to 1.50$)$ & 1.07 (0.78 to 1.47$)$ & 0.96 (0.69 to 1.35$)$ & 0.7172 \\
\hline Women & Q1 (<65.0 g/day) & Q2 (65.0 to $71.8 \mathrm{~g} /$ day) & Q3 (71.9 to $79.9 \mathrm{~g} /$ day) & Q4 ( $\geq 80.0$ g/day) & \\
\hline Cases, n & 154 & 158 & 186 & 207 & \\
\hline Controls, n & 341 & 338 & 310 & 289 & \\
\hline Unadjusted & 1.00 (reference) & 1.04 (0.79 to 1.35$)$ & $1.33(1.02$ to 1.73$)$ & 1.59 (1.22 to 2.06$)$ & $<0.0001$ \\
\hline Model 1 & 1.00 (reference) & 0.95 (0.71 to 1.28$)$ & 1.09 (0.82 to 1.45$)$ & 1.17 (0.88 to 1.55$)$ & 0.1824 \\
\hline Model 2 & 1.00 (reference) & 0.995 (0.74 to 1.34$)$ & 1.09 (0.82 to 1.46$)$ & 1.21 (0.91 to 1.62$)$ & 0.1476 \\
\hline Model 3 & 1.00 (reference) & 0.97 (0.71 to 1.34$)$ & $1.11(0.81$ to 1.51$)$ & 1.19 (0.87 to 1.63$)$ & 0.1830 \\
\hline (b) & Quartile of potassium intake & & & & $P$ for trend \\
\hline Men & Q1 (<2246.2 mEq/day) & Q2 (2246.2 to $2796.1 \mathrm{mEq} /$ day) & Q3 (2796.2 to $3451.1 \mathrm{mEq} /$ day) & Q4 ( $\geq 3451.2$ mEq/day) & \\
\hline Cases, n & 168 & 157 & 146 & 159 & \\
\hline Controls, n & 397 & 408 & 418 & 406 & \\
\hline Unadjusted & 1.00 (reference) & $0.91(0.70$ to 1.18$)$ & $0.83(0.64$ to 1.07$)$ & $0.93(0.72$ to 1.20$)$ & 0.4288 \\
\hline Model 1 & 1.00 (reference) & 0.78 (0.59 to 1.02$)$ & 0.64 (0.49 to 0.84$)$ & 0.63 (0.48 to 0.83$)$ & 0.0005 \\
\hline Model 2 & 1.00 (reference) & 0.84 (0.64 to 1.11$)$ & 0.70 (0.52 to 0.93$)$ & 0.70 (0.52 to 0.94$)$ & 0.0101 \\
\hline Model 3 & 1.00 (reference) & 0.83 (0.62 to 1.12$)$ & 0.64 (0.47 to 0.88$)$ & 0.63 (0.44 to 0.89$)$ & 0.0035 \\
\hline Women & Q1 (<2860.8 mEq/day) & Q2 (2860.8 to 3417.8 mEq/day) & Q3 (3417.9 to $4056.2 \mathrm{mEq} /$ day) & Q4 ( $\geq 4056.3$ mEq/day) & \\
\hline Cases, $\mathrm{n}$ & 191 & 179 & 172 & 163 & \\
\hline Controls, n & 305 & 316 & 325 & 332 & \\
\hline Unadjusted & 1.00 (reference) & 0.91 (0.70 to 1.17$)$ & 0.85 (0.65 to 1.09$)$ & $0.78(0.60$ to 1.02$)$ & 0.0573 \\
\hline Model 1 & 1.00 (reference) & 0.78 (0.59 to 1.04$)$ & 0.71 (0.53 to 0.94) & 0.64 (0.48 to 0.85$)$ & 0.0021 \\
\hline Model 2 & 1.00 (reference) & 0.80 (0.60 to 1.06$)$ & 0.71 (0.53 to 0.95$)$ & $0.67(0.50$ to 0.89$)$ & 0.0050 \\
\hline Model 3 & 1.00 (reference) & 0.83 (0.61 to 1.13$)$ & $0.74(0.54$ to 1.02$)$ & 0.65 (0.46 to 0.92$)$ & 0.0116 \\
\hline
\end{tabular}

Protein and potassium intake is calculated from a food frequency questionnaire and energy adjusted by the residual method

'Controls' means normoalbuminuric cases (ACR $<1.13 \mathrm{mg} / \mathrm{mmol}$ )

Model 1 was adjusted for age (years, continuous); Model 2 was adjusted as for model 1 plus body mass index (kg/m², continuous), physical activity (metabolic equivalent task hour, continuous), smoking status (non-smoker, former smoker, or current smoker), alcohol consumption (<150 g, $150-299 \mathrm{~g}, 300-449 \mathrm{~g},>450 \mathrm{~g}$ ethanol/week), energy intake (kcal/day, quartiles); and Model 3 was adjusted as for model 2 plus eGFR (ml/min/1.73 m², continuous), dietary salt intake (g/day, continuous), pulse pressure ( $\mathrm{mmHg}$, continuous), diabetes (yes or no), and hypertention (yes or no)

In model 2 and model 3, the number of subjects to be analyzed decreased by 4 men and 3 women due to missing values for physical activity 
Characteristics of the subjects including dietary content according to the quartile of NEAP are presented in Table 1. Higher NEAP was associated with higher ACR in both men and women, younger age, current smoker, greater alcohol consumption, and higher diastolic blood pressure in men, and with less total physical activity and hypertension in women. Regarding energy-adjusted nutrient intake, higher NEAP was associated with higher protein, in particular higher animal protein, and less plant protein, carbohydrate, potassium, calcium, magnesium, phosphorus, and salt intake in both men and women. Of the major food groups, fish, meat, grain, and egg intake was positively associated with higher NEAP, and vegetable and fruit intake was negatively associated with higher NEAP in both men and women.

The association between NEAP quartile and three grades of albuminuria, microalbuminuria, high normoalbuminuria, and high normoalbuminuria or microalbuminuria, was analyzed (Table 2). First, regarding for the presence of microalbuminuria versus controls (ACR $<1.13 \mathrm{mmol} / \mathrm{mg}$ ) $(n=4242)$, higher NEAP quartile was associated with higher odds ratio in men $(P$ for trend $=0.0130)$ and women ( $P$ for trend $=0.0014$ ) in the fully adjusted model (as Model 3 in Table 2-a). Second, comparable multivariate logistic regression analysis was carried out in subjects without microalbuminuria $(n=5349)$. Regarding the presence of high normoalbuminuria, higher NEAP quartile was associated with higher odds ratio in women $(P$ for trend $=0.0163$ ), but not men ( $P$ for trend $=0.2391$ ) (Model 3 in Table 2-b). Third, regarding the presence of high normoalbuminuria or microalbuminuria, higher NEAP quartile was associated with higher odds ratio in men $(P$ for trend $=0.0407)$ and women ( $P$ for trend $=0.0028$ ), (as Model 3 in Table 2-c). Similar analysis was performed using PRAL as an alternative. The fully adjusted odds ratio (Model 3) for the presence of microalbuminuria, high normoalbuminuria, and high normoalbuminuria or microalbuminuria comparing the lowest to the highest PRAL quartile was 1.39 (95\% CI: 1.03-1.90, $P$ for trend $=0.0338), 1.16$ (95\% CI: 0.91-1.49, $P$ for trend $=0.2375)$, and 1.24 (95\% CI: $0.99-1.55, P$ for trend $=0.055)$ in men, and was 1.48 (95\% CI: $1.06-2.07, P$ for trend $=0.003), 1.34$ (95\% CI: $1.05-1.72, P$ for trend $=$ 0.0116 ), and 1.37 (95\% CI: $1.09-1.72, P$ for trend $=0.0049$ ) in women, respectively.

In terms of analysis of the association between nutrient components associated with NEAP and albuminuria, similar multivariate logistic analyses were implemented to calculate the adjusted odds ratios for microalbuminuria according to protein or potassium quartile (Table 3). Protein intake was not associated with microalbuminuria in either men or women, and neither animal protein intake nor plant protein intake was associated with microalbuminuria (data not shown). In contrast, a higher quartile of potassium intake was associated with a lower odds ratio for microalbuminuria. The adjusted odds ratio for the presence of high normoalbuminuria or microalbuminuria when comparing the lowest and highest potassium quartiles was 0.73 (95\% CI: $0.57-0.94, P$ for trend $=0.0094)$ in men and 0.75 (95\% CI: $0.59-0.95, P$ for trend $=0.0304$ ) in women; protein intake, including animal and plant protein, had no significantly lower or higher adjusted odds ratio for this grade of albuminuria.

\section{Discussion}

This study showed the association between dietary acid load and albuminuria as previously reported; Banerjee et al. reported the association between estimated net acid excretion, using net acid excretion (calculated using PRAL and organic acids) derived from estimated nutrient intake data based on dietary recall questionnaire, and albuminuria based on cross-sectional data from the National Health and Nutrition Examination Survey (NHANES) 1999-2004 in adults in the US [4]. They also reported the association between high dietary acid load using net acid excretion and progression to end-stage renal disease (ESRD) among adult CKD patients with albuminuria [25]. From the Jackson Heart Study, conducted in a community-based AfricanAmerican population in the US, higher net acid excretion derived from FFQ information was independently associated with the presence of microalbuminuria [5]. Regarding the association between NEAP and albuminuria, the Atherosclerosis Risk in Communities study, a communitybased observational study in middle-aged adults in the US, reported that higher NEAP was associated with incident CKD [26]. Also, other studies from East Asia found that higher NEAP was associated with CKD progression in elderly populations $[6,14]$.

However, it should be noted that these previous studies defined albuminuria as ACR $\geq 3.89 \mathrm{mg} / \mathrm{mmol}$, a cutoff value that is higher than that in our study $(1.13 \mathrm{mg} /$ mmol). The findings of the present study reveal the association between increasing NEAP and high normoalbuminuria in women. A similar but weaker association was seen in men. Also, compared with women, men had lower albuminuria and tended to have hypertension and diabetes. This suggests a sex difference in the association between increasing NEAP and high normoalbuminuria. A novel finding of our study was the association between estimated dietary acid load and high normoalbuminuria, and to our knowledge, this study is the first report of such an association. The clinical relevance of high normoalbuminuria has been suggested because it could lead to adverse outcomes such as cardiovascular disease, ESRD, and all-cause death in the general population [8, 9 , 27]. Furthermore, for primary prevention of albuminuria, it is important to identify lifestyle-factors associated with high normoalbuminuria or microalbuminuria. 
We investigated the nutrients related to NEAP and found that potassium was an important dietary component for the association between NEAP and albuminuria, but protein was not. Some other studies reported an association of potassium intake with CKD. Ko et al. reported an association between dietary acid load and CKD in community-dwelling elderly Koreans $(n=1369$, aged $\geq 65$ years) and that potassium intake was associated with CKD, but protein intake was not [6]. Additionally, another Japanese study $(n=217)$ reported that potassium excretion, but not protein, in 24-h urine samples was a significant component of NEAP [14]. Although the results of these studies were similar to the present study finding in terms of nutrients and NEAP, the present study is complemented with a larger-scale compared with these previous studies. Also, these findings from East Asian countries may suggest that the role of potassium in the association of NEAP with CKD including albuminuria depends on food preference. Because fruits and vegetables are major sources of potassium, some studies have reported their significance $[4,28]$. An interventional study confirmed that kidney injury decreased following reduced acid load in humans, and that acid load reduction by consumption of fruits and vegetables decreased kidney injury markers, including urine albumin concentration, in hypertensive CKD patients with low eGFR (eGFR $60-89 \mathrm{ml} / \mathrm{min} / 1.73$ $\mathrm{m}^{2}$ or eGFR $15-29 \mathrm{ml} / \mathrm{min} / 1.73 \mathrm{~m}^{2}$ ) [28]. The NHANES III study also found that high consumption of fruits and vegetables was inversely associated with albuminuria [4].

In terms of acid-base balance, protein contributes to acid production due to the content of sulfur-containing amino acids. According to Remer et al. evaluating the acid-forming potential of more than 100 frequently consumed foods and beverages by assessing PRAL revealed that animal protein was a source of higher acid production than plant protein [21]. In the present study, total protein intake and animal protein intake was positively associated with increasing NEAP and plant protein intake was inversely associated with increasing NEAP (Table 1); however, the association between animal or plant protein intake and albuminuria was not significant. Although a previous study from the US reported the average American dietary protein resource as being predominantly from animal sources (69\%) [29], the present study showed that the proportion of animal protein in total protein intake was an average of $46.2 \%$ in men and $49.4 \%$ in women. Thus, intake of animal protein might have less impact on the association between NEAP and albuminuria than reported in Western countries.

To our knowledge, there is limited nutritional epidemiologic data aimed at reducing albuminuria. Our findings may indicate that protein restriction has a less important role but potassium has a more important role in individuals, especially East Asians, with high normoalbuminuria or microalbuminuria. However, an important note is that our study did not provide evidence on the safety limit for potassium or protein intake.

Our study has some limitations. First, ACR was measured only once and this might cause misclassification of albuminuria and misunderstanding as to whether there would be chronicity. Furthermore, there was no information as to the type of antihypertensive medication such as renin-angiotensin system blockers. Thus, the effect of drugs which would possibly affect the degree of albuminuria could not be fully eliminated. Second, measurements of plasma $\mathrm{pH}$ or $\mathrm{HCO}_{3}$ which are indices of the degree of acidemia were not available. Third, dietary acid load was estimated using NEAP from self-reported FFQ information only. Dietary contents could be assessed by only a semiquantitative evaluation. In addition, our FFQ was validated by comparing a 12-day weighed food record in men and women aged 40-74 years $(n=240)$ [20], but the subjects in the present study ranged in age from 40 years up to 97 years old. This difference in validation might cause misclassification of exposures and could weaken the strength of association. Fourth, because of the cross-sectional observational study design, a causal relationship between dietary acid load and albuminuria cannot be ascertained. Fifth, the subjects were from the Uonuma area of Japan, and so these findings may not be generalizable to other East Asian populations or other ethnicities worldwide.

\section{Conclusion}

In conclusion, our study has shown that higher dietary acid load was associated with the presence of not only microalbuminuria but also high normoalbuminuria in an adult Japanese population. Regarding the nutrients associated with dietary acid load, potassium intake was negatively associated with the early stages of albuminuria. Longitudinal studies are needed to confirm whether dietary acid load influences the development and progression of albuminuria.

\section{Abbreviations}

95\% Cl: 95\% confidence interval; ACR: Urine albumin-to-creatinine ratio; BMI: Body mass index; CKD: Chronic kidney disease; eGFR: Estimated glomerular filtration rate; ESRD: End-stage renal disease; FFQ: Food frequency questionnaire; HbA1c: Glycated hemoglobin; IQR: Interquartile range; NEAP: Net endogenous acid production; NHANE: National Health and Nutrition Examination Survey; PRAL: Potential renal acid load

\section{Acknowledgements \\ The Uonuma CKD cohort study is based on the Uonuma cohort and Yuzawa cohort study. We thank all the study participants and all members of the Uonuma and Yuzawa cohort study group. We also thank the participating institutions of Niigata Prefecture, Minamiuonuma City, Uonuma City, and Yuzawa Town for their cooperation.}

\section{Funding}

This study was supported by funding from Niigata Prefecture and in part from the Japan Society for the Promotion of Science KAKENHI (Grant No. 17 K15850). The funding source had no role in the study design and findings. 


\section{Availability of data and materials}

The analysis dataset for the current study is available from the corresponding author on reasonable request.

\section{Author's contributions}

$\mathrm{KK}, \mathrm{MH}, \mathrm{RT}, \mathrm{KN}, \mathrm{YI}, \mathrm{NS}, \mathrm{AS}, \mathrm{ST}, \mathrm{JT}$, and IN made substantial contributions to the conception and design of the study, data collection, or interpretation of the data. KK, RT, KN, and NS contributed to the data analysis. KK, MH, RT, KN, $\mathrm{YI}, \mathrm{NS}, \mathrm{AS}, \mathrm{ST}$, and IN assisted in drafting the article or critically revising it for important intellectual content. Each author has sufficiently participated in relevant aspects of the work to take responsibility for this manuscript. All authors reviewed and approved the final manuscript.

\section{Ethics approval and consent to participate}

The study protocol was reviewed and approved by the Ethics Committee of Niigata University (approval numbers 2012-1640, 2015-2140, 2017-0054). Written informed consent was obtained from participants. The study adhered to the tenets of the Declaration of Helsinki.

\section{Consent for publication}

Not applicable

\section{Competing interests}

The authors declare that they have no competing interests.

\section{Publisher's Note}

Springer Nature remains neutral with regard to jurisdictional claims in published maps and institutional affiliations.

\section{Author details}

'Department of Health Promotion Medicine, Niigata University Graduate School of Medical and Dental Sciences, 1-757 Asahimachi-dori, Chuo-ku, Niigata 951-8510, Japan. ${ }^{2}$ Department of Clinical Nutrition Science, Niigata University Graduate School of Medical and Dental Sciences, Niigata, Japan. ${ }^{3}$ Department of Food Science and Nutrition, Nara Women's University Graduate School of Humanities and Sciences, Nara, Japan. ${ }^{4}$ Division of Preventive Medicine, Niigata University Graduate School of Medical and Dental Sciences, Niigata, Japan. ${ }^{5}$ Department of Applied Molecular Medicine, Kidney Research Center, Niigata University Graduate School of Medical and Dental Sciences, Niigata, Japan. ${ }^{6}$ Epidemiology and Prevention Group, Center for Public Health Sciences, National Cancer Center, Tokyo, Japan. ${ }^{7}$ Division of Cliniacal Nephrology and Rheumatology, Niigata University Graduate School of Medical and Dental Sciences, Niigata, Japan.

\section{Received: 15 February 2019 Accepted: 23 April 2019}

\section{Published online: 30 May 2019}

\section{References}

1. Nath KA, Hostetter MK, Hostetter TH. Pathophysiology of chronic tubulointerstitial disease in rats. Interactions of dietary acid load, ammonia, and complement component C3. J Clin Invest. 1985;76:667-75.

2. Scialla JJ, Anderson CA. Dietary acid load: a novel nutritional target in chronic kidney disease? Adv Chronic Kidney Dis. 2013;20:141-9.

3. Adeva MM, Souto G. Diet-induced metabolic acidosis. Clin Nutr. 2011;30:416-21.

4. Banerjee T, Crews DC, Wesson DE, et al. Dietary acid load and chronic kidney disease among adults in the United States. BMC Nephrol. 2014;15:137.

5. Banerjee T, Tucker K, Griswold M, et al. Dietary potential renal acid load and risk of albuminuria and reduced kidney function in the Jackson heart study. J Ren Nutr. 2018;28:251-8.

6. Ko BJ, Chang Y, Ryu S, et al. Dietary acid load and chronic kidney disease in elderly adults: protein and potassium intake. PLoS One. 2017;12:e0185069.

7. Hillege HL, Fidler V, Diercks GF, et al. Urinary albumin excretion predicts cardiovascular and noncardiovascular mortality in general population. Circulation. 2002;106:1777-82.

8. Chronic Kidney Disease Prognosis Consortium, Matsushita K, van der Velde $\mathrm{M}$, et al. Association of estimated glomerular filtration rate and albuminuria with all-cause and cardiovascular mortality in general population cohorts: a collaborative meta-analysis. Lancet. 2010;375:2073-81.

9. Tanaka F, Komi R, Makita S, et al. Low-grade albuminuria and incidence of cardiovascular disease and all-cause mortality in nondiabetic and normotensive individuals. J Hypertens. 2016;34:506-12.
10. Frassetto LA, Todd KM, Morris RC Jr, et al. Estimation of net endogenous noncarbonic acid production in humans from diet potassium and protein contents. Am J Clin Nutr. 1998;68:576-83.

11. Scialla JJ, Appel LJ, Astor BC, et al. Estimated net endogenous acid production and serum bicarbonate in African Americans with chronic kidney disease. Clin J Am Soc Nephrol. 2011;6:1526-32.

12. Remer T, Manz F. Estimation of the renal net acid excretion by adults consuming diets containing variable amounts of protein. Am J Clin Nutr. 1994;59:1356-61.

13. Gennari FJ, Hood VL, Greene T, et al. Effect of dietary protein intake on serum total $\mathrm{CO} 2$ concentration in chronic kidney disease: modification of diet in renal disease study findings. Clin J Am Soc Nephrol. 2006;1:52-7.

14. Kanda E, Ai M, Kuriyama R, et al. Dietary acid intake and kidney disease progression in the elderly. Am J Nephrol. 2014;39:145-52.

15. Kabasawa K, Tanaka J, Nakamura K, et al. Study design and baseline profiles of participants in the Uonuma CKD cohort study in Niigata, Japan. J Epidemiol. 2019; in press.

16. Matsuo S, Imai E, Horio M. Collaborators developing the Japanese equation for estimated GFR, et al: revised equations for estimated GFR from serum creatinine in Japan. Am J Kidney Dis. 2009;53:982-92.

17. Whitworth JA, World Health Organization. International Society of Hypertension Writing Group. 2003 World Health Organization (WHO)/ International Society of Hypertension (ISH) statement on management of hypertension. J Hypertens. 2003;21:1983-92.

18. Levey AS, Coresh J. Chronic kidney disease. Lancet. 2012;379:165-80.

19. Sawada N, Iwasaki M, Yamaji T, et al. The Japan public health center-based prospective study for the Next generation (JPHC-NEXT): study design and participants. J Epidemiol. 2019; doi: 10.2188.

20. Yokoyama Y, Takachi R, Ishihara J. Validity of short and long selfadministered food frequency questionnaires in ranking dietary intake in middle-aged and elderly Japanese in the Japan public health center-based prospective study for the Next generation (JPHC-NEXT) protocol area. J Epidemiol. 2016;26:420-32.

21. Remer T, Manz F. Potential renal acid load of foods and its influence on urine pH. J Am Diet Assoc. 1995;95:791-7.

22. Willett W, Stampfer MJ. Total energy intake: implications for epidemiologic analyses. Am J Epidemiol. 1986;124:17-27.

23. Remer T, Dimitriou T, Manz F. Dietary potential renal acid load and renal net acid excretion in healthy, free-living children and adolescents. Am J Clin Nutr. 2003;77:1255-60.

24. Department of Health Promotion Medicine, Niigata University. https://ja-jp. facebook.com/NUHPM/. Accessed 28 Dec 2018. (in Japanese).

25. Banerjee T, Crews DC, Wesson DE, et al. High dietary acid load predicts ESRD among adults with CKD. J Am Soc Nephrol. 2015;26:1693-700.

26. Rebholz CM, Coresh J, Grams ME, et al. Dietary acid load and incident chronic kidney disease: results from the ARIC study. Am J Nephrol. 2015;42:427-35.

27. Lee YJ, Cho S, Kim SR. Association between serum bicarbonate levels and albuminuria in stage 3 and stage 4 chronic kidney disease: a cross-sectional study. Clin Nephrol. 2014;81:405-10.

28. Goraya N, Simoni J, Jo C, et al. Dietary acid reduction with fruits and vegetables or bicarbonate attenuates kidney injury in patients with a moderately reduced glomerular filtration rate due to hypertensive nephropathy. Kidney Int. 2012;81:86-93.

29. Smit E, Nieto FJ, Crespo CJ, et al. Estimates of animal and plant protein intake in US adults: results from the third National Health and nutrition examination survey, 1988-1991. J Am Diet Assoc. 1999;99:813-20.

\section{Ready to submit your research? Choose BMC and benefit from:}

- fast, convenient online submission

- thorough peer review by experienced researchers in your field

- rapid publication on acceptance

- support for research data, including large and complex data types

- gold Open Access which fosters wider collaboration and increased citations

- maximum visibility for your research: over $100 \mathrm{M}$ website views per year

At $\mathrm{BMC}$, research is always in progress.

Learn more biomedcentral.com/submission 